\title{
Factors Associated with Medication Adherence Among Patients with Severe Mental Disorders in China: A Propensity Score Matching Study
}

This article was published in the following Dove Press journal: Patient Preference and Adherence

\author{
Lei Dou ${ }^{1,2}$ \\ Lili $\mathrm{Hu}^{3}$ \\ Nan Zhang ${ }^{1,2,4}$ \\ Henry Cutler iD ${ }^{5}$ \\ Yan Wang (iD) ${ }^{6}$ \\ Shunping Li iD ${ }^{1,2}$ \\ 'School of Health Care Management, \\ Shandong University, Jinan 2500I2, \\ People's Republic of China; ${ }^{2} \mathrm{NHC}$ Key \\ Laboratory of Health Economics and \\ Policy Research (Shandong University), \\ Jinan 2500I2, People's Republic of China; \\ ${ }^{3}$ Shandong Mental Health Center, Jinan, \\ 250000, People's Republic of China; \\ ${ }^{4}$ Shandong Cancer Hospital and Institute, \\ Shandong First Medical University and \\ Shandong Academy of Medical Sciences, \\ Jinan 250II7, People's Republic of China; \\ ${ }^{5}$ Centre for the Health Economy \\ Macquarie University, Sydney, 2109, \\ Australia; ${ }^{6} \mathrm{Health}$ Commission of \\ Shandong Province, Jinan 2500I4, \\ People's Republic of China
}

\begin{abstract}
Objective: The 686 project provides free essential drugs and follow-up for poor SMD patients, with the purpose of improving patients' medication adherence and reducing the occurrence of dangerous behaviors. The objective of this research was to determine the factors that influence medication adherence in patients with severe mental disorders (SMD) and analyze whether the 686 program significantly improved patient medication adherence. Methods: This study was conducted among SMD patients in 686 project and non-project SMD patients in the same community in 11 cities in Shandong Province of China. The data collected included basic patient information and disease treatment information. Medication adherence was divided into good adherence and poor adherence. Propensity score matching (1:1 match) was performed to adjust for differences in baseline characteristics.

Results: One thousand two hundred ninety-two patients receiving free medication assistance policy (686 project group) and 1292 non-policy patients (control group) were analyzed. Patients who received the 686 project group had significantly better medication adherence than the non-policy group patients (92.6\% vs.61.2\%). Older age and consolidation period were associated with poor adherence, and education level was the positive determinant for adherence.
\end{abstract}

Conclusion: The 686 project could improve medication adherence to patients with SMD. Additional research will pay attention to medication adherence of patients who are elderly, in consolidation period and lower education levels. It should strengthen the guidance and supervision of patients' medication and strengthen the health education of family members and patients.

Keywords: medication adherence, severe mental disorders, propensity score matching

\section{Introduction}

Severe mental disorders (SMD) refer to a group of mental illnesses with obvious psychotic symptoms and severely impaired social life, including schizophrenia, paranoid disorder, bipolar disorder, schizoaffective disorder, epileptic mental disorder and intellectual disability with mental disorders in China. ${ }^{1}$ In China, 5.81 million people with SMD had been registered by the end of $2017 .^{2}$ In addition to causing a large proportion of morbidity, SMD are linked with poorer health outcomes and increased mortality. ${ }^{3}$ People with SMD have a 2-3 times higher average mortality compared to the general population, which translates to a 10-20 year reduction in life expectancy. ${ }^{3}$ Most patients with SMD require long-term treatment and care, which seriously affects the quality of life and productivity of the patients
Correspondence: Shunping $\mathrm{Li}$

School of Health Care Management,

Shandong University, Wenhua Xi Road 44, Jinan, Shandong Province 2500I2, People's Republic of China

Tel +86-|3|-8893-4998

Email lishunping@sdu.edu.cn 
and their family members. ${ }^{4}$ The diseases burden is high due to its high incidence, high recurrence rate and high disability rate. ${ }^{5,6}$

Medication is an essential part of the treatment of patient with SMD, both in acute episodes and in longterm management. ${ }^{7}$ Patient's adherence to treatment is important for the effectiveness of the mental health service. ${ }^{8}$ Several studies showed that the relapse rate is significantly lower when patients insist on taking medication. ${ }^{9,10}$ Medication adherence can be defined as the extent to which a patient's medication-taking matches that agreed with the prescriber. ${ }^{11}$

Medication non-adherence in severe mental disorders is an important clinical issue. ${ }^{12}$ Various studies have found that adherence with antipsychotics is low, in some studies even as low as $20 \% .^{13,14}$ Jónsdóttir et al examined an adherence rate of $61.6 \%$ in a sample of outpatients with SMD, with $58.4 \%$ in schizophrenia and $66.3 \%$ in bipolar disorder, using blood-level measurements. ${ }^{12}$ Non-adherence to prescribed psychoactive medication greatly increases the risk of illness exacerbation and rehospitalization, ${ }^{8,15}$ and attempted suicide, as well as lower quality of life. ${ }^{16}$ In addition, patients' extreme behaviors, crimes, and accidents make their caregivers to experience many negative emotions such as anxiety and depression, which has seriously affected their quality of life, physical and mental health, and social functions. ${ }^{17}$ Therefore, the repercussions affect not only the individuals, but also the society. ${ }^{18}$

Previous studies indicated that medication adherence with SMD was associated with various factors, such as patient-related, medication-related, illness-related, and external/environment factors. ${ }^{18,19}$ Specifically including age, ${ }^{20}$ insight, $^{21}$ education status, ${ }^{22}$ the number of medications, ${ }^{23}$ side effects of medications, ${ }^{24}$ psychotic symptoms, social support ${ }^{25}$ and therapeutic alliance. ${ }^{26}$ In recent years, some studies have begun to pay attention to the influencing factors of SMD medication adherence in China, ${ }^{27-30}$ but these studies were more concerned with the impact of a certain factor, such as the patient management method, ${ }^{29}$ caregiver related knowledge and behavior level. ${ }^{28}$ It requires systematic research and design of the influencing factors.

It is crucial to provide equitable access to comprehensive health services for people with SMD. ${ }^{6}$ Chinese government has taken a series of actions in the past decade to address the disparities in health care access and provision for people with SMD. For example, the New Healthcare
Reform Plan 2009 incorporated major mental disorders into the public health care scheme. ${ }^{31}$ In 2015, severe mental disorders were included in the coverage of major medical insurance for new rural cooperative medical insurance and urban residents. In 2004 the central government subsidizes local health funds for severe mental disorders management treatment project (686 project) was launched. The target of 686 project was patients with SMD who had a clear diagnosis, and poor patients who lived at home and whose annual household income was lower than the local average annual income level. Psychiatrists provided free and essential medicines regularly according to the disease. Doctors in township health centers or community health service centers followed up the patients regularly, checked their mental status and physical diseases, and conducted risk assessments. The purpose of implementing the 686 project was to improve the cure rate and quality of life of patients, help patients to restore social function, reduce the risk behavior of patients. ${ }^{32}$

Non-adherence is considered a multi-causal phenomenon, it is important to understand factors associated with medication adherence among people with SMD. Increasing the rate of medication adherence and reducing the incidence of dangerous behaviors in SMD patients is one of the main goals of the 686 project. Therefore, the purposes of this study were to determine the factors that influence medication adherence in patients with SMD, and analyze whether the 686 program significantly improved patient medication adherence.

\section{Patients and Methods \\ Design and Study Setting}

This was a retrospective cross-sectional study which was a part of a larger study "The central government subsidizes local health funds for severe mental disorders management treatment project" (686 project) evaluation of implementation effect in Shandong Province. A flow diagram describing the subject recruitment and exclusions is shown in Figure 1 . We randomly selected 11 cities out of 17 cities in Shandong Province and surveyed all patients with SMD in 686 project from July to December, 2017.

\section{Participants}

The inclusion criteria were patients with SMD who have been diagnosed by medical institutions, and enrolled in the mental disorder of the National Information System for Psychosis. Exclusion criteria were those who lived in the 


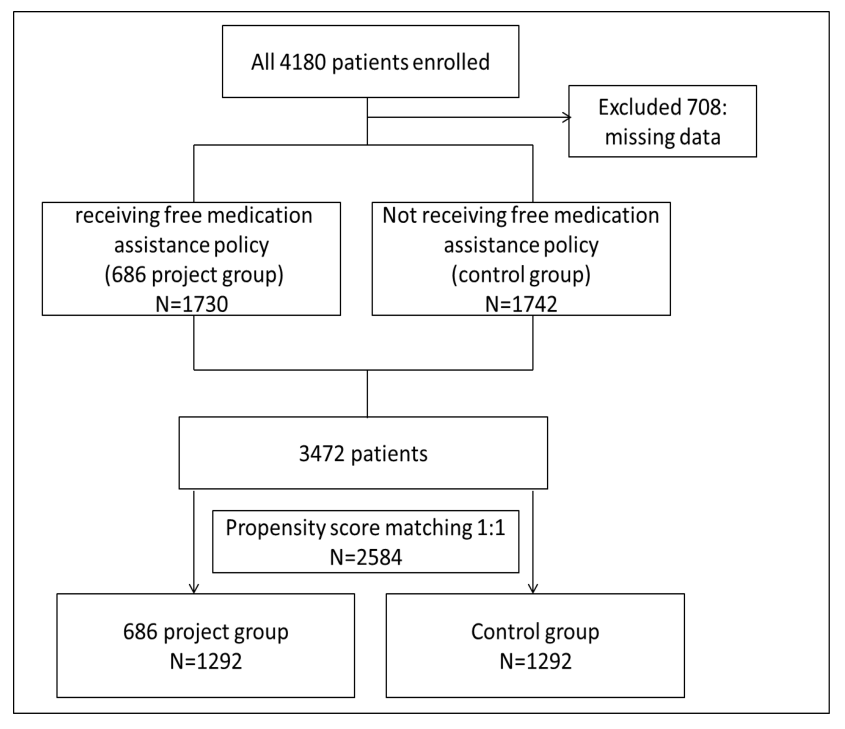

Figure I Flow diagram with a summary of patient recruitment and exclusions. The boxes show the group and number of participants in each process, and arrows indicate the flow of patient recruitment and exclusion.

community for less than 6 months or have no fixed guardian, or those who combined with other serious chronic diseases, such as heart disease, hepatopathy and renal disease or those who cannot follow up the "686 project". Ethical approval (Reference No. ECSHCMSDU20181101) was obtained from the Ethics Review Board of the School of Health Care Management, Shandong University. The patients and guardians provided written informed consent that participation in the study was voluntary and their privacy would be strictly protected. If the participants were illiterate, they could press fingerprint on the informed consent. After that, a questionnaire survey was conducted by their follow-up doctors. If the patient is in recovery period, the questionnaire was answered by the patient; otherwise, the questionnaire was answered by patient's guardian.

The follow-up doctors which were from township health centers or community health service centers checked patient's mental status and physical diseases, and conducted risk assessments, and then completed questionnaires for all patients or their guardians in 686 project, and randomly selected non-project SMD patients in the same community with 1:1 matched based on gender. In the end, excluding 708 questionnaires with incomplete information, the study investigated 1730 patients receiving free medication assistance policy (686 project group) and 1742 non-policy patients (control group).

The patient questionnaire includes basic patient information such as age, gender (male, female), residence (rural, urban), education level (illiterate, primary school, junior high school, high school or technical secondary school, college degree and above), marital status (unmarried, married, divorced/widowed), as well as patient's treatment and follow-up in 2016, stage of disease which was judged by professional doctors and was divided into three: acute period, consolidation period and recovery period. The disease stage was based on the follow-up doctor 's risk assessment of the patient. If the risk is 3-5, the patient is in the acute period, if the risk is $1-2$, the patient is in the consolidation period, and if the risk is 0 , the patient is in the recovery period.

\section{Covariate and Propensity Score Matching (PSM)}

We used a 1:1 propensity score-matched pair method combined with covariate adjustment to analyze patients in two groups shown in Figure 1. The unbalanced conditions at baseline between the two groups were controlled by using PS matching with covariate adjustment. The 1:1 PS matching yielded matched pairs of 1292 patients in policy and 1292 patients non-in policy, resulting in no differences in age, gender, education level, employment status, marriage, family history, annual income, family size and stage of disease.

\section{Intervention}

Patients who were included in the free medication assistance policy would receive free, essential antipsychotic drugs, medication prescriptions were formulated by psychiatrists, and the follow-up doctors would adjust according to the patient's condition. The follow-up doctor supervised the family members of the patient to understand the disease-related knowledge, supervise the patient to take the medicine on time and according to the amount, and regularly take the patient to the hospital for medical examination. Rehabilitation activities and mental health lectures were held regularly in the community, patients and their families were encouraged to participate. Patients who were not included in the policy could not enjoy these services for free.

\section{Medication Adherence Criteria}

Medication adherence was measured with a question "the patient's overall medication use in 2016". This question captured the extent to which patients follow their doctor's advice in regards to medication timing, dosage and 
frequency, and for how long they continued to take their medication compared to the prescribed duration. We set three options to describe this: take medication according to doctor's advice, intermittent medication and non-medication. If the patient takes the medicine according to doctor's advice and does not stop, reduce or increase the dosage of the medicine by himself, it was a complete medication. If the patient reduced the frequency and quantity of the medicine on his own, it was intermittent medication. If the doctor prescribes, but the patient had never used the drug, it was non-medication. If patients choose "take medication according to doctor's advice", it means good adherence while if they choose "intermittent medication or non-medication", it means poor adherence.

\section{Statistical Analysis}

Statistical analysis was performed using SPSS 24.0. Propensity score matching (1:1 match) was performed to adjust for differences in baseline characteristics. The covariates entered into the propensity score were age, sex, education level, employment status, marriage, family history of SMD, annual income, family size and stage of disease.

Baseline characteristics were performed as mean \pm standard deviation (SD). Comparisons between the two groups were performed by using the independent $t$-test for continuous data and chi-square test for categorical data before and after PS matching. Multiple logistic regression approach was used to investigate determinants for medication adherence. The level of significance for independent variables was set at 0.01 .

\section{Results}

\section{Baseline Characteristics Before Propensity Matching}

The baseline characteristics in the pre-match and postmatch cohorts are presented in Table 1. Patients were significantly younger in the 686 project group compared with control group $(44.3 \pm 11.9$ years old versus $47.8 \pm 13.5$ years old), the patient's annual household income in the 686 project group was lower compared with control group (9444 $\pm 10398 ¥$ versus $13376 \pm 19314 ¥)$. There were several parameters of baseline characteristics statistically higher in the 686 project group, including the percentage of male gender $(54.4 \%$ vs $50.4 \%)$, the percentage of junior high school $(38.3 \%$ vs $33.8 \%)$, the percentage of unmarried (35.2\% vs $29.5 \%)$, the percentage of unemployed (14.5\% vs $11.1 \%$ ), the percentage of schizophrenia patients $(86.4 \%$ vs. $79.1 \%)$, and the percentage of unstable disease condition $(3.4 \%$ vs. $1.5 \%)$.

\section{Baseline Characteristics After Propensity Matching}

The matching process eliminated some significant differences that existed between two groups such as gender, age, education level, marital status, employment status, annual household income, and disease condition while the percentage of disease type remained significantly different.

We illustrate histogram of the distribution of the propensity score for both groups before and after propensity matching. Figure $2 \mathrm{~A}$ presents histograms of unbalanced propensity score distribution for both groups before propensity matching. Figure 2B presents histograms of balanced propensity score distribution for both groups after the propensity matching.

\section{Medication Adherence in Patients with SMD}

Figure 3 shows the patient's medication adherence in the 686 project group and the control group. There were $92.6 \%$ of the 686 project group patients were able to take medication according to doctor's advice and regular medication, while only $61.2 \%$ of the control group patients took the drug regularly. In the 686 project group, $7.0 \%$ and $0.4 \%$ of patients were intermittent medication and nonmedication, respectively, compared with $26.5 \%$ and $12.3 \%$ in the control group, respectively.

\section{Factors Associated with SMD Medication Adherence}

The results of both univariate and multivariate logistic regression analysis of the factors affecting patient's medication adherence are shown in Table 2. The univariate results indicated that patient's age, education level and stage of disease were significant predictors of adherence. The multivariate results indicated that factors associated with poor adherence were similar to univariate results, such as being older (OR: $1.01 ; 95 \%$ CI: $1.00-1.02$ ), consolidation period (OR: 1.84;95\% CI: 1.41-2.40). Being more educated was positive determinants for adherence. Sex, living residence, family history of SMD, marital status, annual household income and the number of family members had no statistically significant effect on adherence. 


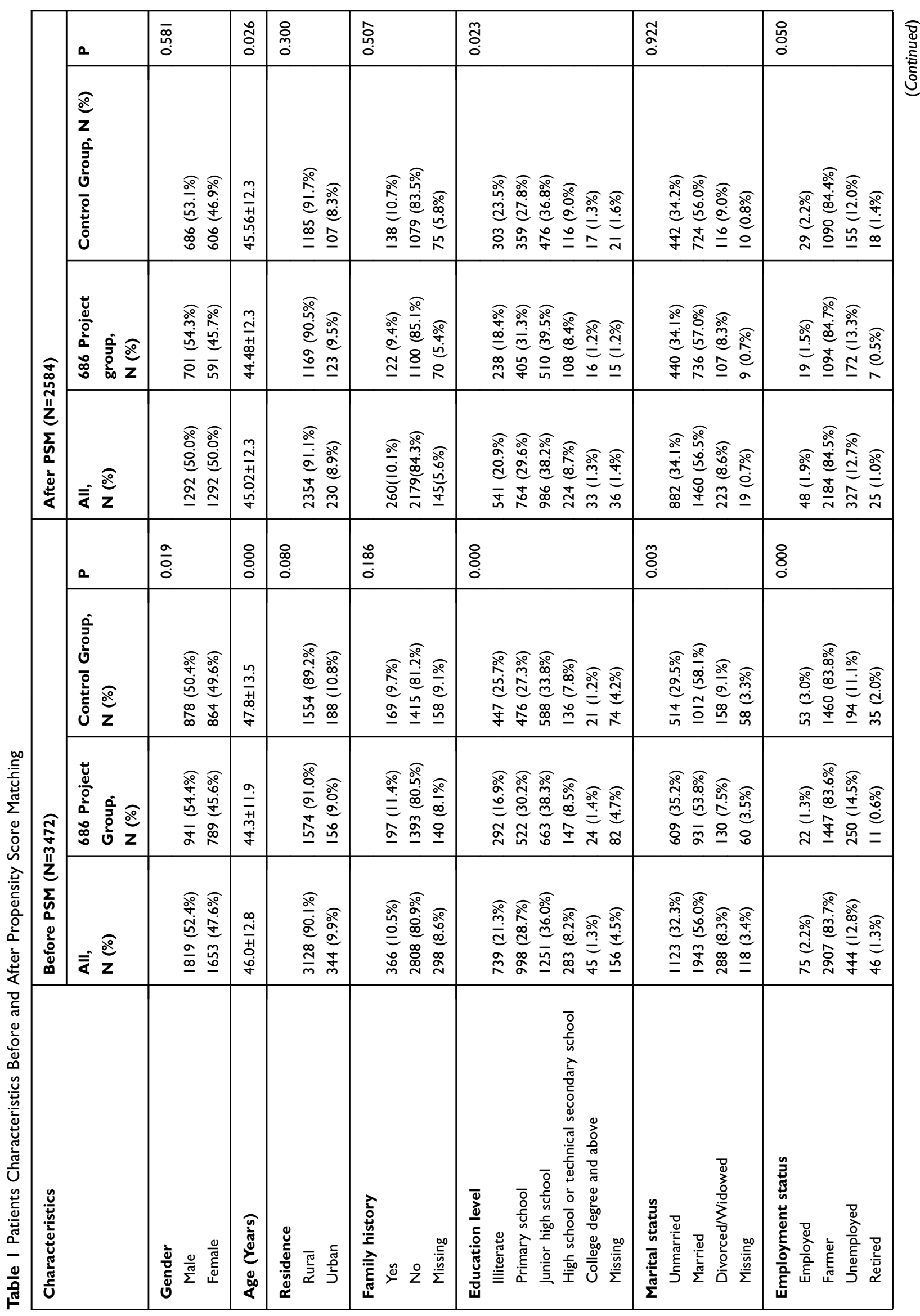




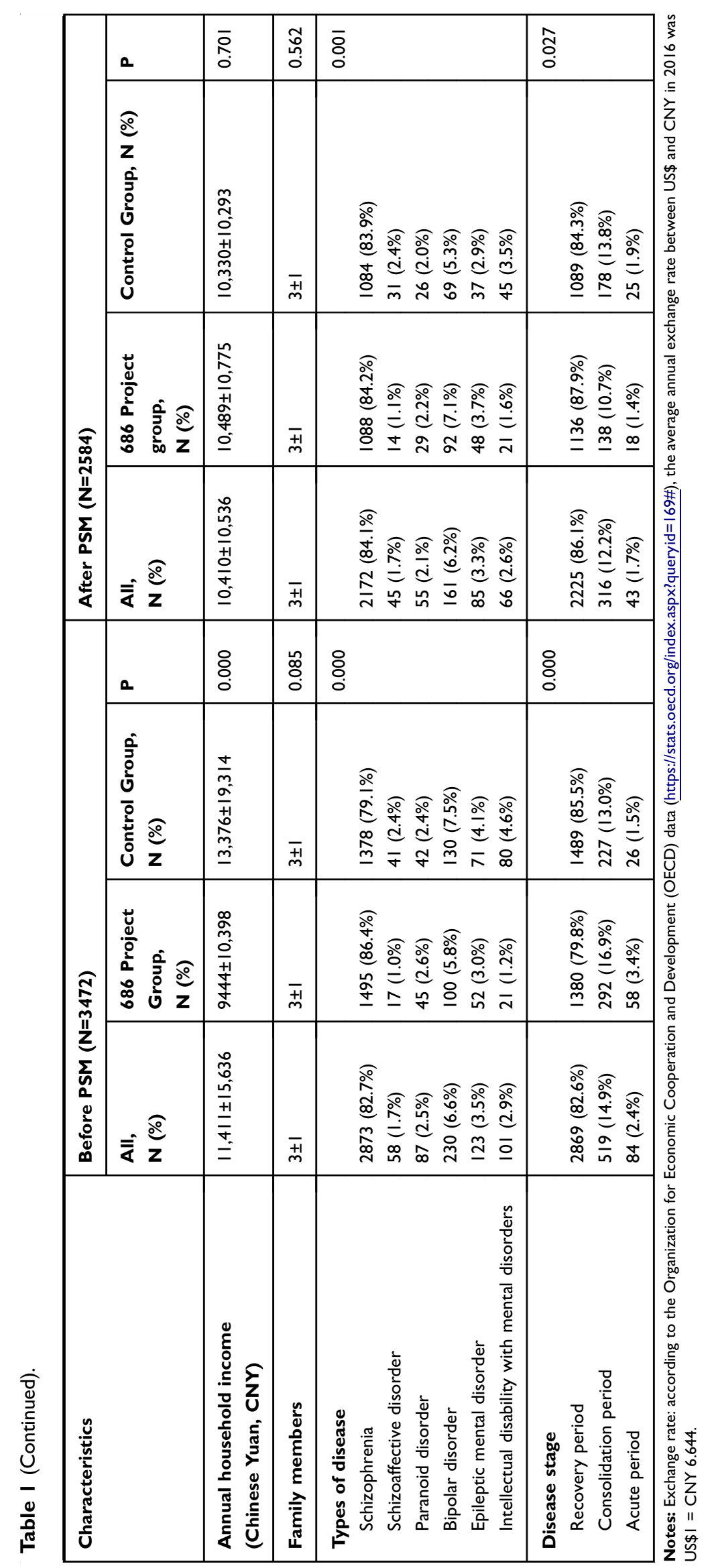


A

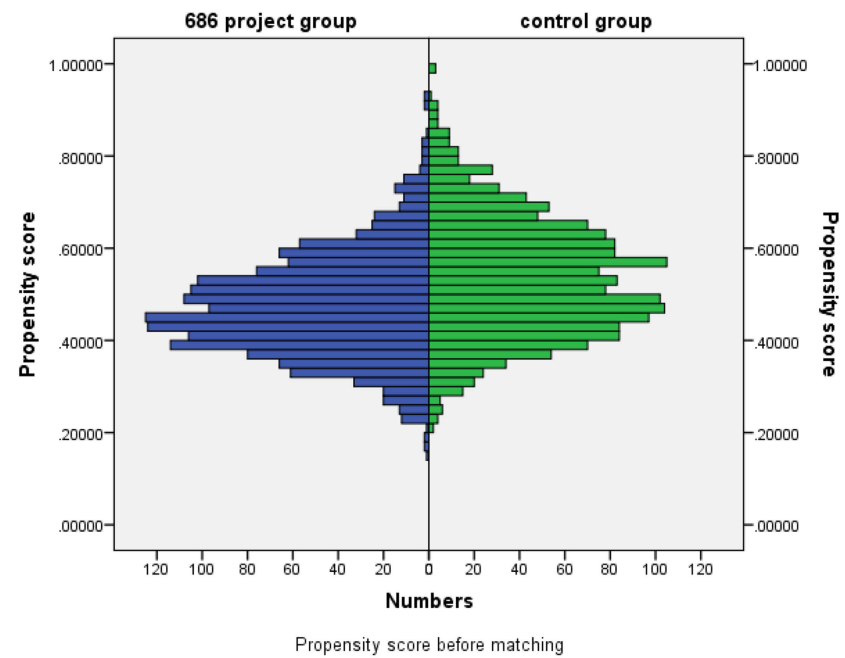

B

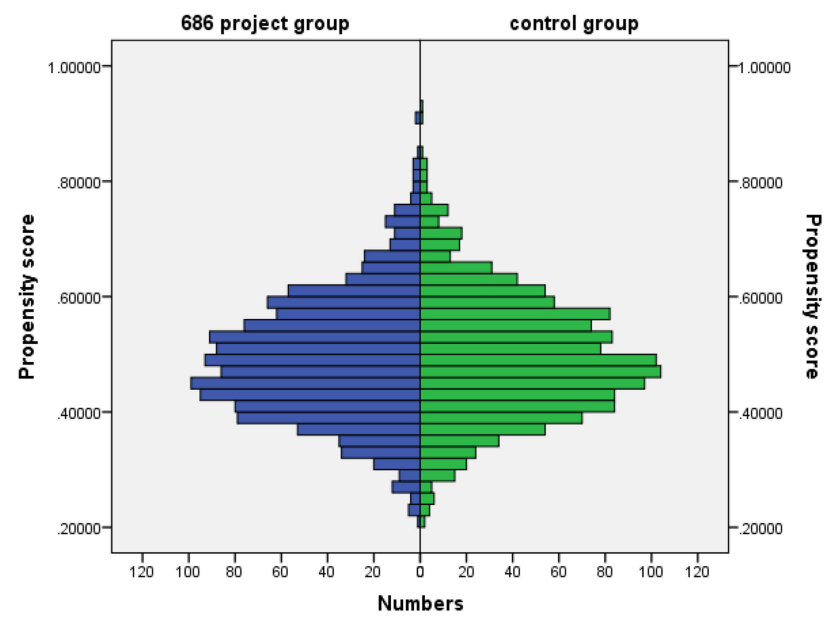

Propensity score after matching

Figure 2 Histograms of propensity score distribution before and after propensity score matching. The propensity scores of participants in the 686 project group are shown with blue histograms, and propensity scores of participants in the control group are shown with green histograms. (A) is histograms of propensity score distribution before propensity score matching. (B) is histograms of propensity score distribution after propensity score matching.

\section{Discussion}

The study applied propensity score matching to minimize confounding effects to evaluate the impact of the 686 project on medication adherence in patients with severe mental disorders and other factors that influence medication adherence. Patients who received the 686 project group had significantly better medication adherence than the non-policy group patients ( $92.6 \%$ vs.61.2\%). We found that older age and consolidation period were associated with poor adherence. Furthermore, education level was the positive determinant for adherence.

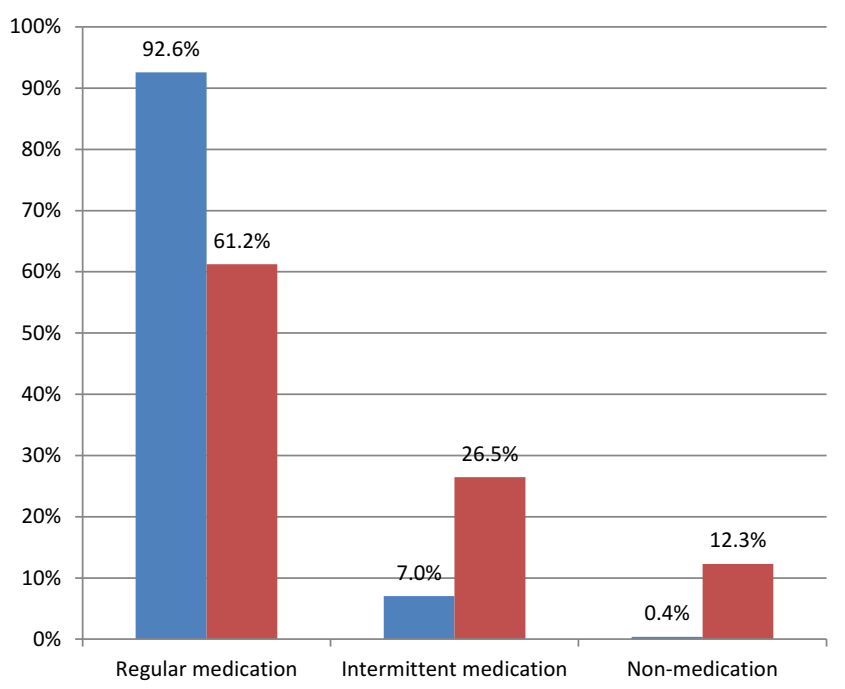

Figure 3 Medication adherence in two group patients. The patient's medication adherence in the 686 project group are shown with blue histograms, and the patient's medication adherence in the control group are shown with red histograms.
The first finding is that the propensity score matching could address the balanced baseline characteristics between the two groups and improve the internal validity of the results. This method has been widely used to reduce bias in recent years. Randomized controlled trials are considered the optimal study design, ${ }^{33}$ but sometimes in retrospective studies when randomization is impractical, unethical, or impossible, nonrandomized observational studies may be useful. ${ }^{34}$ However, we could be faced with inherent indication bias in education level, marital status, employment status and annual household income in the study. Thus, we consider using propensity score matching which is a statistical procedure for reducing this bias by assembling a sample in which observable confounding factors are balanced between treatment groups. ${ }^{34}$

This study found that the medication adherence of patients in the 686 project group was much higher than that of the control group, which indicated that the implementation of the 686 project has a significant effect on improving medication adherence. This result was similar to previous studies that also reported higher adherence in patients with severe mental disorders after receiving the free medication policy. ${ }^{35,36}$ People with SMD often lack access to health services or receive poor quality care, including promotion and prevention, screening, and treatment. ${ }^{37}$ The 686 project works through two channels. First, patients can receive essential drugs for free at the nearest township hospital or community health service 


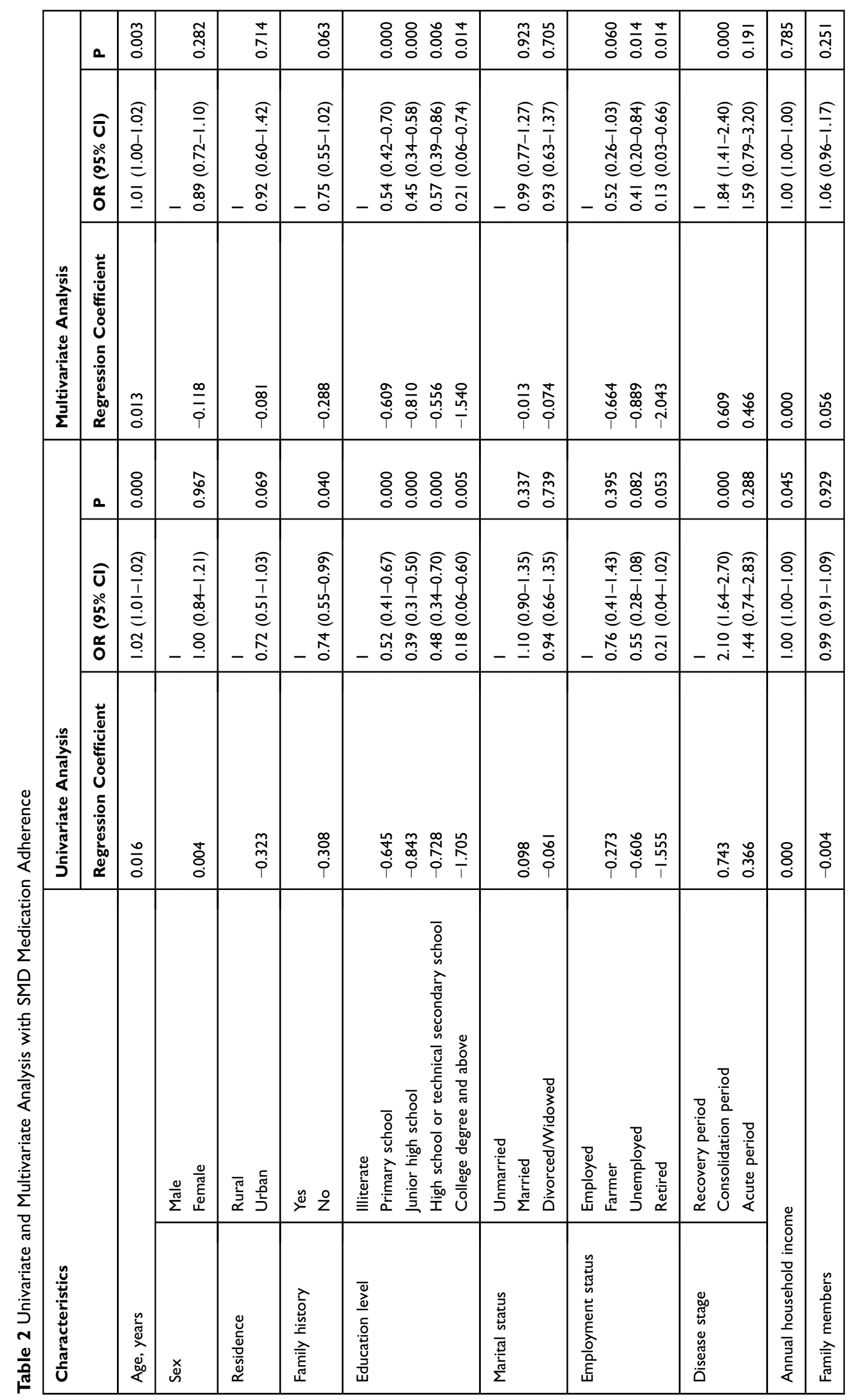


center, ${ }^{38}$ which improves the accessibility and availability of the patient's medication and reduces the economic burden. In addition, the effect of health education on improving medication adherence in patients with mental disorders has been confirmed by many studies. ${ }^{39,40}$ There are communities doctors regularly follow up and hold health lectures to improve the understanding of patients and their families about diseases and medications in 686 project, and effectively improve the medication adherence. $^{41}$

Previous literature review suggested that medication adherence can be classified into four categories: patientrelated, medication-related, illness-related, and external/ environmental factors. ${ }^{19}$ The factors designed in this study are more focused on patient-related factors, including age, sex, residence, family history, education level, marital status, employment status, annual household income, family members and stage of disease. We found that age, education level and stage of disease were significantly associated with medication adherence.

We found that for every additional year of age, the risk of medication non-adherence increased by 1.01 times. Several previous studies have reported that medication adherence increases with age. ${ }^{21,22}$ A study in Australia mentioned that older patients were more likely to be non-adherent. ${ }^{24}$ It might be related to the higher prevalence of comorbidity such as hypertension, diabetics, and other chronic diseases in older people. Hence, they are often prescribed multiple treatments and pill burden could lead to non-adherence to their medications. ${ }^{22,42}$ Furthermore, older patients could fail to adhere to treatments because of cognitive deficit, ${ }^{43}$ including working memory loss and impaired executive performance. ${ }^{43}$ Several studies reported that age had no association with adherence, ${ }^{44,45}$ however, other studies discovered that age was positively correlated with adherence, ${ }^{20,23}$ with the possible reason was older patients having more experience in the course of the disease, which led them to be more compliant with medication. ${ }^{46}$ Compared to cross-sectional studies, larger longitudinal studies need to design to confirm the correlation between age and medication adherence in psychiatric disorders.

Education level was another demographic characteristic related to adherence, which was similar to prior research. Johnson et al observed patients with more education are more adherent than patients with less education in patients with self-reported bipolar disorder. ${ }^{47}$ Linden et al found that patients with worsened adherence had significantly lower school qualifications than others. ${ }^{48}$ It is possible that educational level will affect patients' awareness and attitude towards disease, as well as confidence in medication and their desire to return to society. ${ }^{49}$ As a result, they may be more willing to comply with the medication regimen.

Interestingly, we also found that patients in consolidation period were the least medication adherence comparing with patients in acute and recovery period. Patients with SMD are not able to make a correct estimate of their mental state due to psychotic symptoms such as hallucinations and delusions, and have no self-insight. ${ }^{50}$ For patients in recovery period, their psychotic symptoms basically disappear and self-insight is relatively high, ${ }^{51}$ so they can take medication according to doctor's advice. For patients in acute period, they will be more cared for by family members and the number of doctor's follow-up visits will be increased, which will prompt him to take the medicine on time. ${ }^{52}$ The social function of the patients during the consolidation period basically recovered, which causing the patients and their families to thought that their condition was cured, and they would stop taking the medicine on their own initiative. ${ }^{53}$

Although we have made several interesting findings, the present study also has several limitations. First, propensity score matching can only control for observed covariates such as sex, age, residence, education level, marital status et al in the study. However, any unobserved covariates (used drug, patient cognition and social support) cannot be adjusted to balancing baseline characteristics between two groups. As a result, statistical inferences may still be subject to bias from unmeasured confounding variables. ${ }^{54}$ Second, it was a retrospective study, all information comes from the memories of patients and their families. However, due to the long duration of illness in some patients, there may be recall bias in the information. In the future, the survey time can be shortened to three months or six months to reduce information bias and recall bias. Third, the study did not use a scale to measure patient adherence, such as Medication Adherence Questionnaire (MAQ) and the 8-item Morisky Medication Adherence Scale (MMAS), although we used three questions to classify adherence. But these selfreported adherence rates may overestimate the prevalence of adherence.

In conclusion, these findings reinforce the importance of 686 project to improve medication adherence to patients with severe mental disorders, and any future study needs to pay attention to medication adherence of patients who are elderly, in consolidation period and lower education levels. In the 686 project, it is also necessary to continue to 
strengthen the guidance and supervision of patients' medication and strengthen the health education of family members and patients in order to consolidate the treatment effect and improve the quality of life.

\section{Ethics Approval}

The study was approved by the Ethics Review Board of the School of Health Care Management, Shandong University (Reference No.ECSHCMSDU20181101), and the research adhered to tenets of the Declaration of Helsinki.

\section{Funding}

This study was supported by the Social Science Planning Research Project of Shandong Province (No. 18CZKJ21).

\section{Disclosure}

The authors declare that they have no conflicts of interest.

\section{References}

1. China DCAP. Standards for management and treatment of severe mental disorders (2018 edition). Available from: http://www.gov.cn/ gongbao/content/2018/content_5338247.htm. Accessed May 20, 2020.

2. Xinhua News Agency. The number of patients with severe mental disorders registered in China reached 5.81 million. Available from: http://www.xinhuanet.com/2018-01/26/c_1122323778.htm. Accessed August 12, 2019.

3. Liu NH, Daumit GL, Dua T, et al. Excess mortality in persons with severe mental disorders: a multilevel intervention framework and priorities for clinical practice, policy and research agendas. World Psychiatry. 2017;16(1):30. doi:10.1002/wps.20384

4. Chen Z. Survey on psychological status and family burden of family members of hospitalized patients with schizophrenia. J Qilu Nurs. 2010;11.

5. Guo CY, Jun LÜ, Wu MF, Wei XH. Management of registered patients with severe mental illness in Jinshan District, Shanghai. Chin J Rehabil Theory Pract. 2018;24(06):734-739.

6. WHO. Guidelines for the Management of Physical Health Conditions in Adults with Severe Mental Disorders. Geneva: World Health Organization; 2018.

7. Stentzel U, Berg NVD, Schulze LN, et al. Predictors of medication adherence among patients with severe psychiatric disorders: findings from the baseline assessment of a randomized controlled trial (Tecla). BMC Psychiatry. 2018;18(1):155. doi:10.1186/s12888-018-1737-4

8. Weiden P, Glazer W. Assessment and treatment selection for "revolving door" inpatients with schizophrenia. Psychiatry Quart. 1997;68 (4):377-392. doi:10.1023/A:1025499131905

9. Hogarty GE, Goldberg SC, Schooler NR. Drug and sociotherapy in the aftercare of schizophrenic patients. III. Adjustment of nonrelapsed patients. Arch Gen Psychiatry. 1974;31(5):609-618. doi:10.1001/archpsyc.1974.01760170011002

10. Loebel A, Lieberman J, Alvir J, Geisler J, Koreen A, Chakos M. Time to treatment response in successive episodes of early onset schizophrenia. Schizophr Res. 1995;15(1-2):158. doi:10.1016/09209964(95)95489-V

11. Peter H, Cecilia B, Jan S. Nonadherence with antipsychotic medication in schizophrenia: challenges and management strategies. Patient Relat Outcome Meas. 2014;5:43.
12. Jónsdóttir H, Opjordsmoen S, Birkenaes AB, et al. Medication adherence in outpatients with severe mental disorders: relation between self-reports and serum level. J Clin Psychopharm. 2010;30(2):169175. doi:10.1097/JCP.0b013e3181d2191e

13. Kampman O, Lehtinen K. Compliance in psychoses. Acta Psychiat Scand. 1999;100(3):167-175. doi:10.1111/j.1600-0447.1999.tb10842.x

14. Ravi L, Jan S. Treatment non-adherence in affective disorders. Acta Psychiat Scand. 2010;105(3):164-172.

15. Law MR, Soumerai SB, Dennis RD, Adams AS. A longitudinal study of medication nonadherence and hospitalization risk in schizophrenia. J Clin Psychiat. 2008;69(1):47. doi:10.4088/JCP.v69n0107

16. Coldham EL, Addington J, Addington D. Medication adherence of individuals with a first episode of psychosis. Acta Psychiat Scand. 2002;106(4):286-290. doi:10.1034/j.1600-0447.2002.02437.x

17. Veltman A, Cameron JI, Stewart DE. The experience of providing care to relatives with chronic mental illness. J Nerv Ment Dis. 2002;190(2):108-114. doi:10.1097/00005053-200202000-00008

18. Tham XC, Xie H, Chng CM, Seah XY, Lopez V, Klainin-Yobas P. Factors affecting medication adherence among adults with schizophrenia: a literature review. Arch Psychiatr Nurs. 2016;30(6):797809. doi:10.1016/j.apnu.2016.07.007

19. Abdelbaki A, Ouelletplamondon C, Malla A. Pharmacotherapy challenges in patients with first-episode psychosis. J Affect Disorders. 2012;138(138 Suppl):S3-S14. doi:10.1016/j.jad.2012.02.029

20. Sweileh WM, Ihbesheh MS, Jarar IS, et al. Antipsychotic medication adherence and satisfaction among Palestinian people with schizophrenia. Curr Clin Pharmacol. 2012;7(1):49-55. doi:10.2174/ 157488412799218761

21. Moritz S, Favrod J, Andreou C, et al. Beyond the usual suspects: positive attitudes towards positive symptoms is associated with medication noncompliance in psychosis. Schizophrenia Bull. 2013;39 (4):917-922. doi:10.1093/schbul/sbs005

22. Tadele E, Amha T, Dagim A, Gebremedhin S, Adissu A. Factors associated with medication adherence among patients with schizophrenia in Mekelle, Northern Ethiopia. PLoS One. 2015;10(3): e120560.

23. Mullins CD, Obeidat NA, Cuffel BJ, Naradzay J, Loebel AD. Risk of discontinuation of atypical antipsychotic agents in the treatment of schizophrenia. Schizophr Res. 2008;98(1):8-15. doi:10.1016/j. schres.2007.04.035

24. Mccann TV, Lu S. Medication adherence and significant others' support of consumers with schizophrenia in Australia. Nurs Health Sci. 2009;11(3):228-234. doi:10.1111/j.1442-2018.2009.00440.x

25. Yang J, Ko YH, Paik JW, et al. Symptom severity and attitudes toward medication: impacts on adherence in outpatients with schizophrenia. Schizophr Res. 2012;134(2-3):226-231. doi:10.1016/j. schres.2011.11.008

26. Rungruangsiripan M, Sitthimongkol Y, Maneesriwongul W, Talley S, Vorapongsathorn T. Mediating role of illness representation among social support, therapeutic alliance, experience of medication side effects, and medication adherence in persons with schizophrenia. Arch Psychiat Nurs. 2011;25(4):269-283. doi:10.1016/j. apnu.2010.09.002

27. Zhou Q-H. Clinical investigation and analysis of drug compliance in patients with severe mental disorders in community. Health MustRead Magazine. 2018;(6):290-291.

28. Yang Q-H, Yun Xiao, Zheng KE, Ya-lan Liao. Effect of knowledge and behavioral level of caregivers on medication compliance in patients with severe mental disorders. J Guangdong Med Univ. 2018;36(5):581-584.

29. Li Y-L. Study on the effect of management network of patients with severe mental disorders on medication compliance and recurrence rate. Electron J Clin Med Lit. 2019;6(31):81-82.

30. Cheng X, Yang J-Y, Li D-D, Zhu H-J, Tong Y-B. Relationship between medication compliance and quality of life in patients with schizophrenia. Guangdong Med J. 2019;40(1):116-119. 
31. Xu J, Wang J, Wimo A, Qiu C. The economic burden of mental disorders in China, 2005-2013: implications for health policy. BMC Psychiatry. 2016;16:137. doi:10.1186/s12888-016-0839-0

32. Good BJ, Good MJ. Significance of the 686 program for China and for global mental health. Shanghai Arch Psychiatry. 2012;24(3):175

33. Jupiter DC. Propensity score matching: retrospective randomization? $J \quad$ Foot Ankle Surg. 2017;56(2):417-420. doi:10.1053/j. jfas.2017.01.013

34. Morgan CJ. Reducing bias using propensity score matching. J Nucl Cardiol. 2017;s12317-s12350.

35. Li Q-T, Song J-W, Zhang W-J, et al. Free medication for severe mental illness in rural communities management. Chin J Health Psychol. 2014;22(10):1494-1496.

36. Yang Q-P, Zhang $\mathrm{H}$. The influence of free medication in community on compliance and relapse of schizophrenia. J Clin Psychiatry. 2011;21(6):404-405.

37. Marc DH, Correll CU, Julio B, et al. Physical illness in patients with severe mental disorders. I. Prevalence, impact of medications and disparities in health care. World Psychiatry. 2011;10(1):52-77.

38. Zhao J-W, Xu Y-H, Pan J-X, et al. The evaluation of free medication policy for psychotic patients in poverty in Shanghai Jiading district. Shanghai Arch Psychiatry. 2006;6:342-343.

39. Qiao Y-S, Zhao S-Q, Cui W. The effect of mental health education diverse family therapy on social and family functioning in patients with schizophrenia. Chin J Health Psychol. 2019;27(2):165-169.

40. Ye Z-J, Zhou X, Liang L-P. Effects of health education on family members on self-awareness and medication compliance of patients with mental illness. Chin J Rural Med Pharm. 2018;25(2):61-62.

41. Han S-F, Li S-F, Li L, Huang J-R, Jiang L, Liu X. Cause analysis and health education of schizophrenia patients stopping drug. Chin J Health Psychol. 2011;19(5):529-530.

42. Taj F, Tanwir M, Aly Z, et al. Factors associated with non-adherence among psychiatric patients at a tertiary care hospital, Karachi, Pakistan: a questionnaire based cross-sectional study. J Pak Med Assoc. 2008;58(8):432-436.

43. Acosta FJ, Hernández JL, Pereira J, Herrera J, Rodríguez CJ. Medication adherence in schizophrenia. World J Psychiatr. 2012;2 (5):74. doi:10.5498/wjp.v2.i5.74
44. Farley JF, Hansen RA, Yuisenberg KS, Maciejewski ML. Antipsychotic adherence and its correlation to health outcomes for chronic comorbid conditions. Prim Care Companion CNS Disord. 2012;14(3).

45. Margeti BA, Jakovljevi M, Ivanec D, Toi G, Margeti B. Novelty seeking and medication adherence in patients with schizophrenia. Psychiat Res. 2011;186(1):143.

46. Maeda K, Kasai K, Watanabe A, Henomatsu K, Rogers MA, Kato N. Effect of subjective reasoning and neurocognition on medication adherence for persons with schizophrenia. Psychiat Serv. 2006;57 (8):1203-1205. doi:10.1176/ps.2006.57.8.1203

47. Johnson F, Ozdemir SR, Hauber A, Burch S, Thompson T. Factors that affect adherence to bipolar disorder treatments: a stated-preference approach. Med Care. 2007;45(6):545-552. doi:10.1097/ MLR.0b013e318040ad90

48. Linden M, Scheel TF. Improvement of patient compliance after switching from conventional neuroleptics to the atypical neuroleptic amisulpride. J Psychopharmacol. 2006;20(6):815-823. doi:10.1177/ 0269881106061154

49. Xiao L, Xie YF. Influencing factors for medication adherence of patients with schizophrenia to paliperidone palmitate and its effect on the recurrence of schizophrenics. Pract Pharm Clin Remedies. 2019;22(03):282-285.

50. Barfar E, Sharifi V, Amini H. Cost-effectiveness analysis of an aftercare service vs treatment-as-usual for patients with severe mental disorders. J Ment Health Policy Econ. 2017;20(3):101.

51. Liu S. Impact of health education on rehabilitation of schizophrenic patients. Health Care Guide. 2019;17:102.

52. Lai H, Li S, Mao C. Investigation on the therapeutic compliance of acute schizophrenic patients with psychotic symptoms and the emotional expression of their family members. West Chin Med J. 2016;31 (5):936-939.

53. Jiang L. Factors affecting the compliance of patients with mental illness during the rehabilitation period and nursing countermeasures medical. J Chin People's Health. 2010;22(2):167-172.

54. Joffe MM, Rosenbaum PR. Invited commentary: propensity scores. Am J Epidemiol. 1999;150(4):327-333. doi:10.1093/oxfordjournals aje.a010011
Patient Preference and Adherence

\section{Publish your work in this journal}

Patient Preference and Adherence is an international, peer-reviewed, open access journal that focuses on the growing importance of patient preference and adherence throughout the therapeutic continuum. Patient satisfaction, acceptability, quality of life, compliance, persistence and their role in developing new therapeutic modalities and compounds to optimize clinical outcomes for existing disease states are major areas of interest for the journal. This journal has been accepted for indexing on PubMed Central. The manuscript management system is completely online and includes a very quick and fair peer-review system, which is all easy to use. Visit http:// www.dovepress.com/testimonials.php to read real quotes from published authors. 